\title{
Simultaneously prediction of sheep and goat carcass composition and body fat depots using in vivo ultrasound measurements and live weight
}

\author{
Luís G. Dias ${ }^{\mathrm{a}}$, Severiano R. Silva ${ }^{\mathrm{b}}$, Alfredo Teixeira ${ }^{\mathrm{a}, *}$ \\ ${ }^{\text {a }}$ CIMO, Instituto Politécnico de Bragança, 5300-253, Portugal \\ ${ }^{\mathrm{b}}$ CECAV, Universidade de Trás-os-Montes e Alto Douro, 5001-801, Portugal
}

\section{A R T I C L E I N F O}

\section{Keywords:}

Sheep breeds

Goat breeds

Carcass composition

Body fat depots

in vivo ultrasound measurements

Predictive models

\begin{abstract}
A B S T R A C T
The present study established multiple linear regression models using two ultrasound in vivo measurements (at lumbar and sternal regions, with different real-time ultrasonography machines and probes) and live weight, to predict simultaneously carcass composition and body fat depots of different breeds of sheep and goat. This study is important for the small ruminant industry, considering the feasibility of using the ultrasound methodology in field conditions, as well as an online system of the carcass evaluation. The multiple linear regression models were obtained by selecting the best subset of variables between using the in vivo measurements (raw variables), their second degree and interactions, evaluated in terms of prediction performance using cross-validation "K-folds" and validated by a test group. Overall, high accuracy $\left(\operatorname{adj~} \mathrm{R}^{2}\right.$ ) was obtained from the linear relationship between predicted and experimental values of the group test for each of the nine dependent variables, with values varying between adj $\mathrm{R}^{2} 0.88$ and 0.98 .
\end{abstract}

\section{Introduction}

The development of precise methods for predicting body and carcass composition in meat animals is of major importance for performance testing, grading and for breeding selection schemes (Bünger et al., 2011; Scholz et al. 2015). In recent years, there have been important developments in non-invasive and non-destructive image techniques to obtain objective data of carcass and body composition (Szabo Cs et al., 1999; Scholz et al. 2015). Among the various image methods available, real-time ultrasonography (RTU) has become the most common technique for body and carcass composition assessment of sheep and goat species (Stouffer 2004; Scholz et al. 2015). Different imaging methods were also used to evaluate carcass composition of meat producing species as the light lamb carcasses by video image analysis (Silva et al. 2014) and light kid carcass by bioelectrical impedance analysis (Silva et al. 2017). Extensive experimental work has been carried out to collect information about the relationship between measurements obtained with RTU and carcass traits of the principal meat animals (Teixeira et al. 2008; Silva et al. 2006; Hopkins et al. 2007; Leeds et al. 2008; Greiner et al. 2003; Moeller and Christian 1998).

The present work falls within the scope of the application of RTU parameters towards prediction of carcass and body composition of meat animals (Thwaites 1984; Teixeira 2008, 2015). Most of the previous works used linear regression models to demonstrate that it was possible to estimate carcass and body composition (Silva et al. 2006; Hopkins et al. 2007; Teixeira et al. 2006, 2008; Ripoll et al. 2010). However, it is recognized that more complex linear and nonlinear models can be applied to successfully estimate, at the same time, several dependent variables (Peres et al. 2010). It is recognized that more complex linear and nonlinear models can be applied to successfully estimate several dependent variables (Font-i-Furnols et al. 2013; Peres et al. 2010). However, there is much to be learned from the use of more adequate models build from RTU measurements to ensure that carcass composition traits can be accurately and consistently predicted to allow solid benefits to the small ruminant meat industry.

The aim of this study was to establish whether two ultrasound in vivo measurements and live weight were adequate to predict simultaneously carcass composition and body fat depots of different breeds of sheep and goat species. This objective has great relevance for the sheep and goat meat industry considering the reduced number of non-invasive measurements made in the live animal; the models were built using data from different breeds of sheep and goats, reared under different production systems in the Iberian Peninsula, enabling their use by technicians as an alternative to reference methods to assess carcass composition and body fat deposits, as the dissection of the carcass, which requires time and is expensive. In general, it will allow to find out a more assertive economic value of the animal or carcass that meets

\footnotetext{
* Corresponding author at: Escola Superior Agrária, Instituto Politécnico de Bragança, Campus de Santa Apolónia, 5300-253 Bragança, Portugal.

E-mail address: teixeira@ipb.pt (A. Teixeira).
} 
Table 1

Overview of the data collected from four databases used in this study.

\begin{tabular}{llll}
\hline Specie & Breed & $\mathrm{n}$ & Reference \\
\hline \multirow{2}{*}{ Sheep } & Rasa Aragonesa & 19 & Delfa et al. (1995) and Ripoll et al. (2009) \\
& Roya Bilbilitana & 22 & Delfa et al. (1995) and Ripoll et al. (2009) \\
& Ojinegra de Teruel & 8 & Delfa et al. (1995) and Ripoll et al. (2009) \\
Goat & Serrana & 20 & Cadavez et al. (2002) \\
& Blanca Celtiberica & 56 & Teixeira et al. (2008) \\
\hline
\end{tabular}

the needs of the meat industry, with the objectivity required by the producer and in accordance with the consumer's requirements.

\section{Materials and methods}

\subsection{Animal datasets}

The database used corresponds to the data of 125 animals from the databases of the works referenced in Table 1, which includes data of carcass composition, body fat depots, total body fat and RTU in vivo measurements of the following sheep and goat breeds: three sheep breeds: Rasa Aragonesa, Roya Bilbilitana and Ojinegra de Teruel (Delfa et al. 1995; Ripoll et al. 2009) and two goat breeds: Serrana goats (Cadavez et al. 2002) and Blanca Celtiberica goats (Teixeira et al. 2008).

\subsection{Live weight and carcass parameters}

The animals were slaughtered after $24 \mathrm{~h}$ fasting under the same conditions and in accordance with European laws. Live weight (LW) was recorded immediately before slaughter. After slaughter, the omental fat (OME), mesenteric fat (MES), and pericardial fat (PER) were removed, weighed and recorded separately. These fat depots were named body fat depots. Carcasses were cooled at $4{ }^{\circ} \mathrm{C}$ for $24 \mathrm{~h}$. After, the carcasses were halved and the left side of each carcass was dissected with a scalpel and the carcass composition was obtained into bone, muscle (MUSC), subcutaneous fat (SF), intermuscular fat (IF), kidney and pelvic fat (KPF), subcutaneous fat tail (SFT) and remainder (major blood vessels, ligaments and tendons). The total body fat (TF) resulted from the sum of the dissected fat depots on carcasses (SF, IF, KPF and SFT) with the body fat depots (OME, MES and PER). The carcass and body fat depots were full described in previous work by Teixeira et al. (1989).

The gathered dataset presents the values for LW, in kilograms, as well as the values of ultrasound measurements, in millimeters, which are the independent variables. Also, as dependent variables in this study, it was recorded the carcass composition tissues, the body fat depots and the total body fat. All those variables are expressed in grams.

\subsection{Ultrasound measurements}

The ultrasound measurements of fat thickness were obtained at lumbar (U_LUMB, ultrasonic fat thickness measurement between the 3rd-4th lumbar vertebra) and sternal (U_STER, ultrasonic fat thickness measurement on 3rd sternebra) regions, with different RTU machines and probes according to the different studies (Cadavez et al. 2002; Delfa et al. 1995; Ripoll et al. 2009; Teixeira et al. 2008). In Ternasco lambs (Rasa Aragonesa, Roya Bilbilitana and Ojinegra de Teruel breeds) and Serrana goats, the fat thickness measurements were taken using a Aloka SSD-500 V equipped with a probe of $7.5 \mathrm{MHz}$ (Cadavez et al. 2002). For Blanca Celtiberica goats, the fat thickness was measured using a Toshiba Sonolayer SAC-32B equipped with a $5 \mathrm{MHz}$ probe (Teixeira et al. 2008). All RTU image capture was performed $24 \mathrm{~h}$ before slaughter.

\subsection{Statistical analysis}

All data processing and statistical methods were performed using the statistical program "open source" $\mathrm{R}$, version 2.15 , with the caret (Kuhn and Johnson 2013), prospectr (Stevens and Ramirez-Lopez 2013), relaimpo (Grömping 2006) and Subselect (Cadima et al. 2004; Cadima et al. 2012) packages.

This study, in an initial exploratory analysis (Maroco 2007), presents the overall values of each animal used in this work (carcass tissues, body fat depots, LW and ultrasound measurements) by analyzing the range values within each different sheep and goat breeds. The following statistical analysis was performed in order to ascertain whether the combined ultrasound in vivo measurements and live weight data were able to predict simultaneously carcass composition and body fat depots of different sheep and goat breeds.

To establish the MLR predictive models, it was considered that the relative growth of fat depots in relation to body or carcass weight in sheep (Teixeira et al. 1989) and goat (Delfa et al. 1994; Teixeira et al. 1995) are not linear. So, the database included the original three variables (LW, U_LUMB and U_STER) as well, the respective squared variables and the possible interactions between them, obtained by mathematical calculation, corresponding to a total of 10 variables. These variables were centered and scaled to avoid the influence of different order of magnitude in variables.

The multiple linear regression (MLR) models were evaluated in terms of the performance of prediction (Maroco 2007), using train group with internal validation (cross-validation "K-folds") and test group. The data from Internal cross-validation "K-folds", root mean squared error (RMSE) and adjusted determination coefficient (Adjusted $\mathrm{R}^{2}$ ), is not shown since their results were similar to the residual standard error (RSE) and Adjusted $\mathrm{R}^{2}$ of the selected models. Multiple linear regression is a supervised technique that allows to obtain a linear equation (the model) between two or more explanatory variables (independent variables) and a response variable (dependent variable). The division of the samples in train group $(60 \%$ of original data to set the model) and test group ( $40 \%$ of original data for external validation) was carried out by the algorithm Kennard-stone, a uniform mapping algorithm (Stevens and Ramirez-Lopez 2013). This algorithm is based in the principal components of the independent variables (two ultrasound in vivo measurements and live weight) and it allowed to ensure that each breed of sheep and goat species were represented in both groups. First, it starts to include in the train group, samples that are farthest apart. Afterward, the remaining variables are selected by stepwise procedure by maximizing the Euclidean distance within samples in the principal components space. To ensure that the best MLR model is obtained, the simulated annealing (SA) algorithm (meta-heuristic variable selection algorithm) was applied to choose the best sub-set of the independent variables that allows to have the highest quality criterion (a function of the standard Wilks' Lambda statistic, which in the context of a univariate linear regression is the coefficient of determination) and lowest RMSE values in the predictive results. To ensure a greater confidence in finding a true optimal solution, 10,000 attempts were used (Cadima et al. 2004; Cadima et al. 2012). The SA variable selection algorithm mimics annealing physic process, where it is intended to have a slow controlled cooling process of a heated material in order to obtain a more stable material, i.e., a structure without defects. Also, it is a metaheuristic algorithm, since it allows to select a subset of the original independent variables (significant variables) within a large search space of other possible subsets of variables, which corresponds to a global optimum for a given approximation criterion (Cadima et al. 2004; Cadima et al. 2012).

To obtain the MLR model, it was used a cross-validation (internal validation) using the procedure K-folds cross-validation, which in this work was a 10-folds application (Kuhn and Johnson 2013).

The model's goodness-of-fit was assessed using RSE and Adjusted $\mathrm{R}^{2}$ values. Since RSE is the average amount that the response will deviate 
from the true regression model, the lower the RSE, the better the model. The Adjusted $\mathrm{R}^{2}$ is the proportion of data variation that is explained by the obtained model, being the better the model whose Adjusted R2 value is the closest to the unit.

The evaluation of the model predictive capacity was made through the values of the slope and intercept (as well as, the respective confidence intervals) plus the RSE and Adjusted $\mathrm{R}^{2}$ obtained from the relationship between the expected experimental and predicted values for the established model using data of train and test group.

In the final MLR model, the significance of the independent variables used in the model was also verified by the level of significance of 0.05 ( $p$-value) and $\mathrm{R}^{2}$ contribution averaged over orderings among regressors (Grömping 2006). In order to visualize that the obtained linear models are validated in their predicting performance (Maroco 2007), it is presented graphically, as example, the results from best and worst cases obtained in this work:

linear relationship between the predicted values obtained by the multiple linear regression model and the expected values of the train group data (including linear adjustment) and test group data;

- dispersion of residuals of test group data in order to verify that the residuals follow any trend or have a random distribution; included is an adjustment line to pass through the data;

- normal Q-Q to evaluate the normality of the standardized residuals of the test group data following the theoretical quantile, to verify if the distribution was normal (represented by the graph line defined);

- Cook distance of each sample of the test group to check whether there was a presence of "outliers" in the test group data, considering that values greater than 1 are indicative that it is excessively influential data in the estimation of the regression coefficients.

\section{Results and discussion}

In this study, data was gathered from several studies (Delfa et al. 1995; Ripoll et al. 2009; Cadavez et al. 2002; Teixeira et al. 2008) in order to incorporate different breeds of two species in the prediction models. The purpose was to confirm whether it was possible to have common predictive models for carcass composition, body fat depots and total body fat using in vivo measurements of those animals. As a result, a database was gathered with 125 animals and in the following sections it is shown the descriptive analysis of each animal breed/species and the evaluation of prediction models using MLR.

\subsection{Descriptive analysis of data}

In Table 2 is shown the range values (minimum and maximum) of LW and ultrasound measurements obtained at lumbar and sternal regions (3 independent variables), as well as the carcass composition, body fat depots and total body fat ( 9 dependent variables) for the different sheep and goat breeds. As can be seen, the Serrana goats had the lowest values of LW, followed by the Rasa Aragonesa, Ojinegra de Teruel and Roya Bilbilitana lambs, whose values are similar and, therefore, forming a mixed a group with a LW range between 20 and $24 \mathrm{~kg}$. The Blanca Celtiberica goats have a wider range of values comprising the higher LW data of the dataset. The same trends are found in the remaining variables as they showed to be highly correlated with the variable LW (generally $R>0.84$, with exceptions of SF and SFT presenting R values equal to 0.75 and 0.67 , respectively; data not shown). The overall data matrix presents wide range of values for all dependent variables and for the three independent variables, LW and thickness measurements of subcutaneous fat in lumbar and sternum regions, suggesting that this is a representative animal sample. The wide variation of data is an essential attribute in the studies aiming the in vivo prediction of carcass composition (Scholz et al. 2015; Silva et al. 2020). Several studies highlighted that the best prediction models are from traits that show a high coefficient of variation (Hopkins et al.
1993; Silva et al. 2006; Teixeira et al. 2008). Those authors report low accuracy in predicting carcass composition traits when used RTU measurements that show reduced variation.

\subsection{MLR models using variable selection algorithm}

Table 3 presents the best subsets of independent variables selected by the SA meta-heuristic algorithm of variable selection for the nine dependent variables, the RSE and adjusted $R^{2}$ values, as well the $R^{2}$ contribution averaged of each independent variable in the obtained model. The results showed that the selected subsets contained 2 to 7 independent variables and that the selected models showed $\mathrm{R}^{2}$ values higher than 0.79 . The simplest model was obtained for the muscle variable, which also corresponds to the model with the best predictive power in cross-validation. All obtained MLR models had included ultrasound measurements, being significant in all models ( $p$-value $<$ 0.05). Table 3 shows that the LW variable has contributions varying between $16.6 \%$ and $62.6 \%$ in the obtained models while the raw ultrasonic measurements vary between $6.5 \%$ and $33.5 \%$. Also, only the predictive models of MUS, OME and MES presented a linear contribution of at least one of the ultrasound variables. These overall results showed that the 3 independent variables bring relevant information to improve prediction of carcass and body components relating to animals of different breeds of sheep and goat.

These results confirm the improvement in the body and carcass composition estimation models using LW and two ultrasound measurements as independent variables in goats (Teixeira et al. 2008; Teixeira et al. 2019) and in lambs (Teixeira et al. 2006; Ripoll et al. 2009), as well as to assess the internal adipose depots in dairy sheep (Afonso et al. 2019).

\subsection{MLR models predictive capacity}

The predictive capacity of the best MLR model obtained, for each dependent variable, was evaluated through the results obtained from the relationship between the expected experimental and model predicted values using the train data and test group: slope, intercept, confidence intervals at 0.05 level, RSE and adjusted $\mathrm{R}^{2}$. In Tables 4 and 5 are shown these results for the train and test group, respectively.

It was found that the model slope was significantly different from 0 ( $p$-values $<0.001$ ) in all the models obtained. The data from train group allowed to establish MLR models with adjusted determination coefficients varying between 0.80 and 0.96 (Table 4), which although acceptable, could be improved if the variables did not show great dispersion mainly on the data for Blanca Celtiberica goats. So, it turns out that, in general, the models obtained with the train group data have slopes close to one (1) in the relationship between the predicted and expected training values by the obtained MLR model, but only in MUSC and TF models, the theoretical slopes are considered in the slope confidence interval. In the case of the intercept values for this relationship, only five dependent variables (MUSC, SF, IF, OME and TF) had confidence intervals containing the zero value.

The robustness of predictive models is evaluated for its ability to predict the values of new samples (test group), which is the main objective of this work, and so it will be given more importance to the results shown in Table 5. So, as can be seen, the adjusted $\mathrm{R}^{2}$ obtained from the linear relationship between experimental values of the group test of each of the nine dependent variables and predicted values for these variables varied between 0.76 and 0.98 . Also, confidence intervals for the slope and intercept contain the theoretical values 1 and zero, respectively, with the exception of variables MUSC, SFT and PER.

The best linear model was the prediction model of muscle mass where it was possible to obtain a linear relationship between the values predicted by multiple linear regression model and expected, whose slope and intercept cannot be considered the theoretical (one and zero, respectively). In this adjusted model, the selection of variables by SA 
Table 2

Minimum and maximum ranges of live weight, ultrasound measurements and carcass composition, body fat depots and total body fat components.

\begin{tabular}{|c|c|c|c|c|c|c|c|c|c|c|}
\hline & \multicolumn{6}{|c|}{ LAMBS } & \multicolumn{4}{|c|}{ GOATS } \\
\hline & \multicolumn{2}{|c|}{ Rasa Aragonesa } & \multicolumn{2}{|c|}{ Ojinegra de Teruel } & \multicolumn{2}{|c|}{ Roya Bilbilitana } & \multicolumn{2}{|c|}{ Serrana } & \multicolumn{2}{|c|}{ Blanca Celtiberica } \\
\hline & Min & Max & Min & Max & Min & Max & Min & Max & Min & Max \\
\hline $\mathrm{LW}, \mathrm{kg}$ & 20.2 & 22.8 & 20.3 & 22.5 & 20.0 & 24.0 & 9.4 & 13.1 & 33.0 & 91.5 \\
\hline U_LUMB, mm & 2 & 5 & 4 & 8 & 2 & 8 & 1 & 2 & 0 & 7 \\
\hline U_STER, mm & 13 & 22 & 18 & 22 & 15 & 22 & 11 & 21 & 8 & 52 \\
\hline \multicolumn{11}{|c|}{ Carcass composition, $\mathrm{g}$} \\
\hline MUSC & 2675 & 3122 & 2567 & 2857 & 2438 & 3145 & 1157 & 1955 & 4197 & 12,500 \\
\hline SF & 204 & 450 & 346 & 567 & 247 & 626 & 34 & 192 & 57 & 5550 \\
\hline IF & 390 & 593 & 442 & 629 & 377 & 677 & 127 & 275 & 231 & 4746 \\
\hline KPF & 54 & 236 & 88 & 211 & 78 & 204 & 42 & 306 & 75 & 3004 \\
\hline SFT & 9 & 34 & 19 & 58 & 17 & 37 & 1 & 6 & 4 & 199 \\
\hline \multicolumn{11}{|c|}{ Body fat depots, $g$} \\
\hline OME & 138 & 306 & 258 & 596 & 176 & 407 & 28 & 230 & 208 & 8134 \\
\hline MES & 165 & 309 & 272 & 393 & 187 & 285 & 70 & 223 & 439 & 3619 \\
\hline PER & 4 & 27 & 10 & 19 & 8 & 43 & 3 & 21 & 30 & 440 \\
\hline \multicolumn{11}{|c|}{ Total body fat, $g$} \\
\hline $\mathrm{TF}$ & 1790 & 2828 & 2409 & 3824 & 2028 & 3503 & 534 & 1683 & 1572 & 38,771 \\
\hline
\end{tabular}

LW -live weight; U_LUMB - lumbar subcutaneous fat depth; U_STER - sternum fat depth.

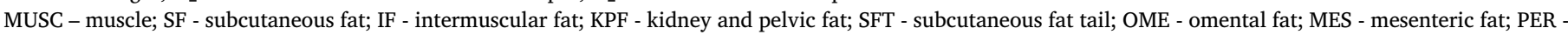
Pericardial fat; TF - total body fat.

Table 3

Independent variables selected by ANNEALING algorithm for each dependent variable.

\begin{tabular}{|c|c|c|c|}
\hline Dependent variable & Independent variables ${ }^{\mathrm{a}}$ & RSE & Adj $R^{2}$ \\
\hline MUSC & LW (62.6\%); U_STER (33.5\%) & 600 & 0.96 \\
\hline SF & $\begin{array}{l}\text { U_LUMB (7.9\%); U_STER (13.8\%); LWxU_LUMB (18.0\%); U_LUMBxU_STER (15.5\%); LWxU_LUMBxU_STER (22.6\%); } \\
\text { U_STER }^{2}(15.1 \%)\end{array}$ & 266 & 0.92 \\
\hline IF & U_STER (19.9\%); LWxU_STER (28.7\%); LWxU_LUMBxU_STER (24.3\%); U_STER ${ }^{2}(20.4 \%)$ & 221 & 0.93 \\
\hline KPF & LWxU_LUMB (38.0\%); LWxU_STER (43.0\%); U_LUMB ${ }^{2}(10.4 \%)$ & 248 & 0.91 \\
\hline SFT & U_STER (16.5\%); LWXU_LUMB (23.5\%); LWxU_LUMBXU_STER (26.1); U_STER ${ }^{2}(17.8 \%)$ & 17 & 0.83 \\
\hline OME & LW (17.4\%); U_LUMB (7.9\%); LWxU_LUMB (21.5\%); LWxU_STER (23.4\%); LWxU_LUMBxU_STER (20.7\%) & 714 & 0.90 \\
\hline MES & U_LUMB (8.1\%); LWxU_LUMB (26.2\%); LWxU_STER (30.3\%); LWxU_LUMBxU_STER (21.7\%) & 389 & 0.86 \\
\hline PER & LW (35.0); U_LUMB (6.5\%); U_LUMBXU_STER (17.8\%); U_STER ${ }^{2}(21.5 \%)$ & 366 & 0.79 \\
\hline TF & LW (16.6\%); U_STER (16.0\%); LWxU_STER (23.0\%); U_LUMBxU_STER (16.2\%); U_LUMB ${ }^{2}(6.4 \%) ;$ U_STER $^{2}(17.0 \%)$ & 0.24 & 0.95 \\
\hline
\end{tabular}

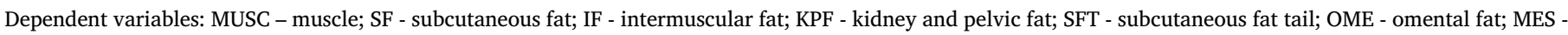
mesenteric fat; PER - Pericardial fat; TF - total body fat.

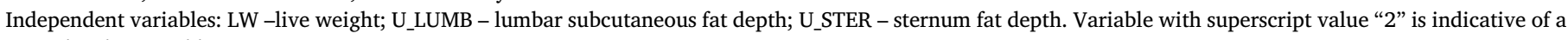
second order variable.

RSE - Residual standard error; Adj R2 - adjusted determination coefficient.

${ }^{\text {a }}$ Within brackets, the $\mathrm{R}^{2}$ contribution, in percentage, averaged of each independent variable in the obtained model.

algorithm, the LW and U_STER, as significant independent variables, explains $98 \%$ of the muscle variability (Table 5). This result shows that it is possible to determine the muscle mass of sheep and goat of different breeds together.
Fig. 1 shows the overall appreciation of the results obtained with the MLR models for the muscle variable in terms of the relationship between the predicted values by the model and the experimental for the test group data, as well the normality of the residuals, according to the

Table 4

Parameters of the linear relationship between the values predicted by the model obtained and the expected for the train group data.

\begin{tabular}{|c|c|c|c|c|c|c|}
\hline \multirow[t]{2}{*}{ Dependent variable } & Parameter & \multirow[t]{2}{*}{ Confidence interval } & \multirow[t]{2}{*}{ Intercept \pm sd } & \multirow[t]{2}{*}{ Confidence interval } & \multirow[t]{2}{*}{ RES } & \multirow[t]{2}{*}{ Adj $R^{2}$} \\
\hline & Slope \pm sd & & & & & \\
\hline MUSC & $0.96 \pm 0.02^{* * *}$ & {$[0.92 ; 1.01]$} & $234 \pm 152$ & {$[-69 ; 537]$} & 584 & 0.96 \\
\hline SF & $0.93 \pm 0.03^{* * *}$ & {$[0.87 ; 0.99]$} & $62 \pm 39$ & {$[-16 ; 139]$} & 248 & 0.93 \\
\hline IF & $0.93 \pm 0.03^{* * *}$ & {$[0.87 ; 0.99]$} & $73 \pm 40^{\times}$ & {$[-6 ; 153]$} & 209 & 0.93 \\
\hline $\mathrm{KPF}$ & $0.91 \pm 0.03^{* * *}$ & {$[0.85 ; 0,98]$} & $94 \pm 45^{*}$ & {$[5 ; 184]$} & 234 & 0.91 \\
\hline SFT & $0.84 \pm 0.04 * * *$ & {$[0.75 ; 0.92]$} & $7 \pm 3 *$ & {$[2 ; 12]$} & 15 & 0.84 \\
\hline OME & $0.91 \pm 0.03^{* * *}$ & {$[0.84 ; 0.98]$} & $195 \pm 105^{\times}$ & {$[-15 ; 404]$} & 662 & 0.91 \\
\hline MES & $0.86 \pm 0.04^{* * *}$ & {$[0.78 ; 0.94]$} & $171 \pm 65^{*}$ & {$[42 ; 300]$} & 354 & 0.86 \\
\hline PER & $0.80 \pm 0.05^{* * *}$ & {$[0.70 ; 0.89]$} & $23 \pm 7^{* * *}$ & {$[9 ; 37]$} & 334 & 0.80 \\
\hline $\mathrm{TF}$ & $0.95 \pm 0.02^{* * *}$ & {$[0.90 ; 1.00]$} & $408 \pm 294$ & {$[-178 ; 993]$} & 0.22 & 0.95 \\
\hline
\end{tabular}

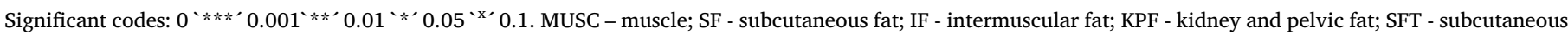
fat tail; OME - omental fat; MES - mesenteric fat; PER - Pericardial fat; TF - total body fat; sd - standard deviation; RSE - Residual standard error; Adj $\mathrm{R}^{2}$ - adjusted determination coefficient. 
Table 5

Parameters of the linear relationship between the values predicted by the model obtained and the expected for the test group data.

\begin{tabular}{|c|c|c|c|c|c|c|}
\hline \multirow[t]{2}{*}{ Dependent variable } & Parameter & \multirow[t]{2}{*}{ Confidence interval } & \multirow[t]{2}{*}{ Intercept \pm sd } & \multirow[t]{2}{*}{ Confidence interval } & \multirow[t]{2}{*}{ RES } & \multirow[t]{2}{*}{ Adj $R^{2}$} \\
\hline & Slope \pm sd & & & & & \\
\hline MUSC & $0.94 \pm 0.02^{* * *}$ & {$[0.90 ; 0.97]$} & $218 \pm 62^{* * *}$ & {$[93 ; 343]$} & 225 & 0.98 \\
\hline $\mathrm{SF}$ & $0.95 \pm 0.05^{* * *}$ & {$[0.85 ; 1.05]$} & $-12 \pm 25$ & {$[-63 ; 39]$} & 109 & 0.88 \\
\hline IF & $0.95 \pm 0,04 * * *$ & {$[0.87 ; 1.03]$} & $-11 \pm 26$ & {$[-63 ; 40]$} & 102 & 0.92 \\
\hline $\mathrm{KPF}$ & $0.93 \pm 0.04^{* * *}$ & {$[0.85 ; 1.00]$} & $-4 \pm 24$ & {$[-53 ; 44]$} & 96 & 0.93 \\
\hline SFT & $0.52 \pm 0.04^{* * *}$ & {$[0.44 ; 0.61]$} & $9 \pm 1^{* * *}$ & {$[6 ; 12]$} & 7 & 0.76 \\
\hline OME & $1.07 \pm 0.04 * * *$ & {$[0.99 ; 1.15]$} & $-22 \pm 39$ & {$[-101 ; 56]$} & 239 & 0.94 \\
\hline MES & $0.98 \pm 0.05^{* * *}$ & {$[0.89 ; 1.07]$} & $28 \pm 29$ & {$[-29 ; 85]$} & 160 & 0.91 \\
\hline PER & $0.78 \pm 0.04^{* * *}$ & {$[0.69 ; 0.86]$} & $12 \pm 3^{* * *}$ & {$[6 ; 17]$} & 192 & 0.88 \\
\hline $\mathrm{TF}$ & $1.04 \pm 0.04 * * *$ & {$[0.97 ; 1.11]$} & $-2 \pm 164$ & {$[-331 ; 328]$} & 0.20 & 0.94 \\
\hline
\end{tabular}

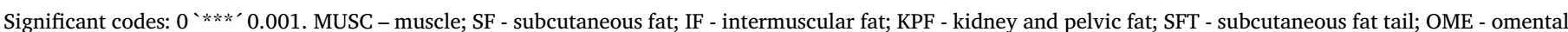

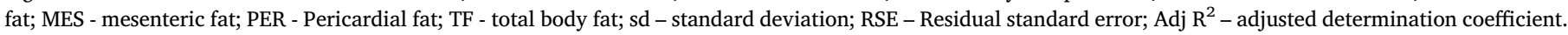

predicted value and according to Cook distance. Observing the Fig. 1.A, the degree of association between variables and the tendency of variation together follows a clear linear tendency. In respect of residuals according to the predicted value (Fig. 1.B), there is an independence of residuals (residues are distributed on a random basis around zero), so, the magnitude of a residue does not influence the magnitude of next residue. In the Fig. 1.C (Normal Q-Q), the values represented are distributed more or less on the main diagonal suggesting a normal distribution of errors. From the Fig. 1.D, the information from Cook distance allowed to infer that within the observations there was the presence of an outlier, a G_BC sample with the lowest muscle mass (sample 3 in the test group data), showing that this experimental muscle quantity is not in accordance to the experimental in vivo measures. Even so, the obtained prediction model is acceptable to provide muscle mass through the animal's measure in vivo. Overall, it was also found the presence of one outlier in the test data group results in more 4 dependent variables (SF, KPF, OME and PER) and three outliers in the MES variable. An outlier in 50 samples of the test data group did not bring major changes to the predictive results, except for the variable MES, which worsened.

The models with the lowest predictive performances were obtained for the SFT and PER variables, with a low slope value of 0.52 and 0.78 , respectively, and an adjusted $\mathrm{R}^{2}$ of 0.76 and 0.88 , respectively, for the linear relation between experimental values and predicted values. With regard to the SFT dependent variable, the overall appreciation of the model obtained, and its predictive capability is presented in Fig. 2. As can be seen in Fig. 2.A, there is a huge data variability that explains the lower performance of the obtained MLR model. This variability can be justified by the low weight of this parameter in each different animal breed and species, which increases the experimental error in its measurement. This situation was also found for the PER variable. Observing the other representations in the Fig. 2, it is shown that, even so, the model data showed independence of residuals, normal distribution of errors and not very influential values in the estimation of the regression coefficients.

Regarding the RSE results obtained in the model fitting (Table 3) and in the linear relationship between the real values and predicted by the model for the training data (Table 4), they are considered high, but acceptable. It is possible to see in Figs. 1 and 2 that in the training group there are some points that deviate from the adjusted models, justifying the results of RSE and adjusted $\mathrm{R}^{2}$.

Also, in the study of predictive capability of the MLR model using the test group data, there was no evidence of the presence of outliers, which contributed to smaller RSE results, demonstrating acceptable predictive performance of the adjusted models.

The muscle mass prediction accuracy results (percentage relative error, $\mathrm{RE} \%$ ) are presented in Table 6 , showing the minimum and maximum values of each subset of specie/breed. As can be seen, the prediction accuracy is acceptable as expected, since in Fig. 1.A, the test group predicted muscle mass values are close to the adjusted line defined by the train group data. Moreover, it was verified that the RE\% values varied between 0.1 and $16.2 \%$, with only $6 \%$ of the samples with values higher than $15 \%$. The other dependent variables showed expected wider intervals, considering their lower fitting adjusted $\mathrm{R}^{2}$ values.

The overall results showed that the multiple linear regression models obtained can be used in predicting body content and fat depots and muscle of new animals. The database allowed having high data amplitude favoring the study of the applicability of the prediction models based on body weights and two ultrasound measurements. The results corroborate the fact that the independent variables, live weight and ultrasound measurements, allow to obtain multiple linear regression models with acceptable results prediction using data from two species of animals and five different breeds since, in general, the adjusted $\mathrm{R}^{2}$ values are higher than 0.87 except for the model subcutaneous fat tail with an adjusted $\mathrm{R}^{2}$ of 0.76 .

This work results from the fusion of multiple data groups used in previous works, which focused on the same topic but using data for different animal breed and species (Delfa et al. 1995; Ripoll et al. 2009; Cadavez et al. 2002; Teixeira et al. 2008) and, due to the small number of data, to obtain estimation models. Due to the broader data matrix, it allowed to evaluate the MLR models in its predictive capacity of the dependent variables in new data. Also, the MLR models were obtained by selecting the best subset of variables between using three in vivo measurements (raw variables), their second degree and interactions, and evaluated using the adjusted determination coefficient (determination coefficient with a correction of the number of samples).

Although a detailed comparison of the estimation and prediction results of the obtained MLR models is not possible since different fitting procedures were used in relation to those reported in other research studies of ultrasound application in the quantification of muscle and fat tissue in carcass/live animals, a comparison to other works was carried out using the coefficients of determination obtained from the relation using model's estimation/prediction data and the expected data. Three works that presented estimation models of several measurements of carcass composition and body fat depots and high accuracy values were considered for modeling results comparison: Silva et al. (2006), which presented $R^{2}$ values varying between 0.68 and 0.99 for in vivo estimation of sheep (native Churra da Terra Quente breed) carcass composition (muscle, subcutaneous fat, inter-muscular fat, internal fat and total fat weights); Ribeiro et al. (2008) that achieved $\mathrm{R}^{2}$ values between 0.89 and 0.97 , in models to estimate internal body fat (carcass kidney fat depth, carcass KPH weight, and internal fat) of cattle (Angus steers); and, Orman et al. (2010) that estimated the carcass traits (carcass subcutaneous fat thickness, carcass LM area, cold carcass weight, carcass yield) of Awassi lambs, showing $\mathrm{R}^{2}$ values ranging from 0.17 to 0.90. Lastly, the work of Lambe et al. (2010) presented the prediction of carcass composition and tissue distribution in beef cattle of crossbred 

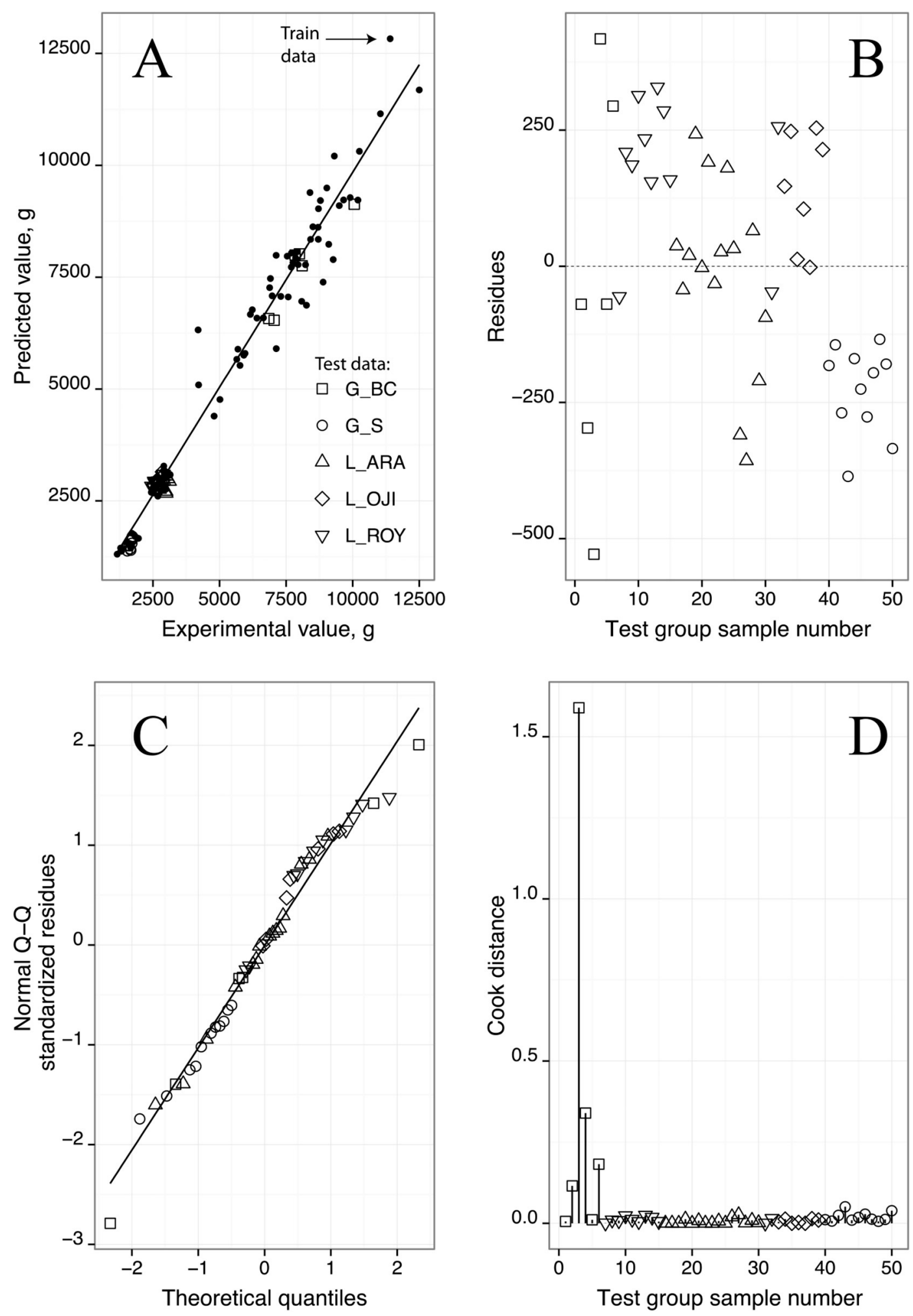

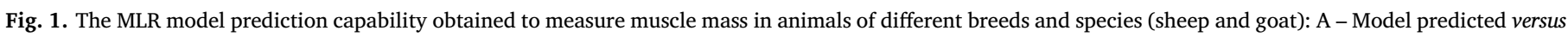

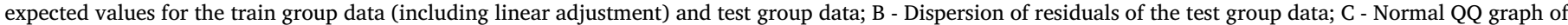
standardized residues of the test group data following the theoretical quantile; D - Cook distance of each sample of the test group.

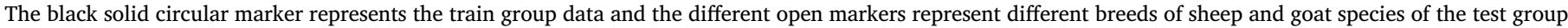
data: G_BC -Blanca Celtiberica goat; G_S- Serrana goat; L_ARA -Rasa Aragonesa lamb; L_OJI -Ojinegra de Teruel lamb; L_ROY -Roya Bilbilitana lamb.

steers and heiferstat. In this work, data was divided into train group, to establish the mathematical models, and test group for validation, to evaluate the model's prediction performance. For this last data, the best results of the relation between predicted and expected values had prediction accuracies $\left(\mathrm{R}^{2}\right)$ between 0.52 and 0.64 for fat, muscle weights and fat proportion in the carcass side.

Overall, these results showed that the estimation models obtained in the present work had adjusted $\mathrm{R}^{2}$ values similar or higher to the $\mathrm{R}^{2}$ 

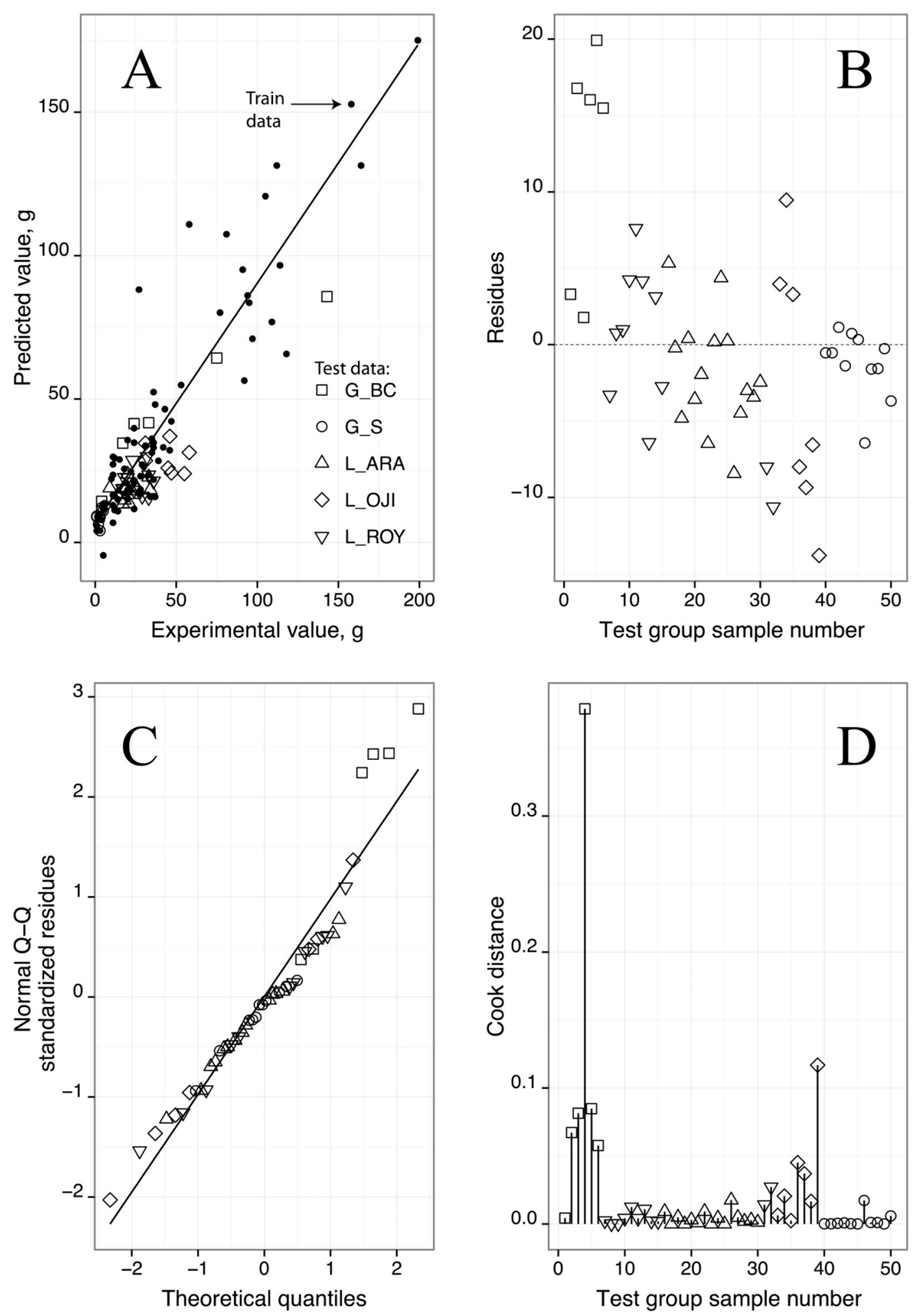

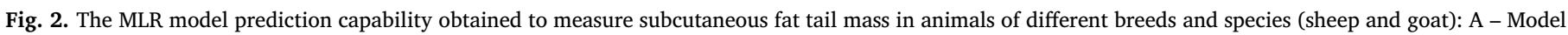
predicted versus expected values for the train group data (including linear adjustment) and test group data; B - Dispersion of residuals of the test group data; C Normal QQ graph of standardized residues of the test group data following the theoretical quantile; D - Cook distance of each sample of the test group. The black solid circular marker represents the train group data and the different open markers represent different breeds of sheep and goat species of the test group data: G_BC -Blanca Celtiberica goat; G_S- Serrana goat; L_ARA -Rasa Aragonesa lamb; L_OJI -Ojinegra de Teruel lamb; L_ROY -Roya Bilbilitana lamb.

values obtained in the works mentioned above but, it is possible to verify that the methodology applied in this work showed high performance adjustment, which can be inferred from the high accuracy (generally, higher than 0.88 ) obtained in the models' validations.
However, in future work other nonlinear regression multivariate techniques should also be tested to improve the data fit and prediction performance. 
Table 6

Muscle mass prediction accuracy obtained in the subsets of species/breed of the test group data.

\begin{tabular}{llll}
\hline & \multicolumn{2}{l}{ Accuracy (RE\%) } & \\
\cline { 2 - 4 } & $\mathrm{n}$ & Min & Max \\
\hline Lambs: & 15 & & \\
Rasa Aragonesa & 7 & 0.1 & 11.1 \\
Ojinegra de Teruel & 11 & 1.8 & 11.6 \\
Roya Bilbilitana & & 0.2 & 15.6 \\
Goats: & 11 & & 1.3 \\
Serrana & 6 & 0.2 & 9.2 \\
Blanca Celtiberica & & & \\
\hline
\end{tabular}

$\mathrm{RE} \%$ - percentage relative error; $\mathrm{n}$ - number of samples; Min - minimum RE\% value; Max - maximum $\mathrm{RE} \%$ value.

\section{Conclusions}

In this study was shown that it was possible to use live weight and two real-time ultrasonography measurements (non-invasive technique) taken in live animals, to get common models to predict body and carcass components for different goat and sheep breeds. These results allow to infer that there is possibility of more extensive applications using the proposed methodology, since these predictive models are open to more data from other different production systems of goat and sheep breeds, in order to increase their robustness and accuracy. The advantages of this methodology are evident because, it is inexpensive; easy to obtain in field conditions; and, by considering that robust prediction models were obtained even using ultrasound data from different real-time ultrasonography machines and probes. Overall, these predictive models can support the assessment of body composition in vivo and carcass classification, which meets the needs of the sheep and goat industry for an objective system of animal evaluation, carcass grading and classification recognized by producers, slaughterhouses, retailers and according to the consumer requirements.

\section{Declaration of Competing Interest}

None.

\section{Acknowledgements}

The authors are grateful to Laboratory of Carcass and Meat Quality of Agriculture School of Polytechnic Institute of Bragança 'Cantinho do Alfredo'. The authors are members of the CYTED network HEALTHY MEAT (119RT0568).

\section{References}

Afonso, J., Guedes, C., Teixeira, A., Santos, V., Azevedo, J., Silva, S., 2019. Using realtime ultrasound for in vivo assessment of carcass and internal adipose depots of dairy sheep. J. Agric. Sci. 157 (7-8), 650-658.

Bünger, L., Macfarlane, J.M., Lambe, N.R., Conington, J., McLean, K.A., Moore, K. Glasbey, C.A., Simm, G., 2011. Use of X-ray computed tomography (CT). In 'UK sheep production and breeding. In: Karuppasamy, S. (Ed.), CT scanning - techniques and applications. INTECH Europe, Rijeka, Croatia, pp. 329-348.

Cadavez, V., Rodrigues, S., Pereira, E., Delfa, R., Teixeira, A., 2002. Predicción de la composición de la canal de cabritos por ultrasonografia in vivo. ITEA 98A. 1. pp. $39-50$.

Cadima, J., Cerdeira, J.O., Minhoto, M., 2004. Computational aspects of algorithms for variable selection in the context of principal components. Comput. Stat. Data Anal 47, 225-236.

Cadima, J., Cerdeira, J.O., Silva, P.D., Minhoto, M., 2012. The subselect R package [Online]. Available at: http://cran.r-project.org/web/packages/subselect/vignettes/ subselect.pdf.

Delfa, R., Teixeira, A., González, C., Gosálvez, L.F., Tor, M., 1994. Relationships between body fat depots, carcass composition, live weight and body condition scores in Blanca Celtibérica goats. Options Méditerranéennes - Série Séminaires 27, 109-120.

Delfa, R., Teixeira, A., González, C., Blasco, I., 1995. Ultrasonic estimates of fat thickness and longissimus dorsi muscle depth for predicting carcass composition of live Aragon lambs. Small Rumin. Res. 16, 159-164.
Font-i-Furnols, M., Brun, A., Tous, N., Gispert, M., 2013. Use of linear regression and partial least square regression to predict intramuscular fat of pig loin computed tomography images. Chemom. Intel. Lab. Syst. 122, 58-64.

Greiner, S.P., Rouse, G.H., Wilson, D.E., Cundiff, L.V., Wheeler, T.L., 2003. The relationship between ultrasound measurements and carcass fat thickness and longissimus muscle area in beef cattle. J. Anim. Sci. 81, 676-682.

Grömping, U., 2006. Relative importance for linear regression in R: the package relaimpo. J. Stat. Softw. 17 (1), 1-27.

Hopkins, D.L., Pirlot, K.L., Roberts, A.H.K., Beattie, A.S., 1993. Changes in fat depths and muscle dimensions in growing lambs as measured by real-time ultrasound. Aust. J. Exp. Agric. 33, 707-712.

Hopkins, D.L., Stanley, D.F., Ponnampalam, E.N., 2007. Relationship between real-time ultrasound and carcass measures and composition in heavy sheep. Aust. J. Exp. Agric. 47, 1304-1308.

Kuhn, M., Johnson, K., 2013. Applied Predictive Modeling. Springer Science + Business Media, New York, USA.

Lambe, N.R., Ross, D.W., Navajas, E.A., Hyslop, J.J., Prieto, N., Craigie, C., Bünger, L., Simm, G., Roehe, R., 2010. The prediction of carcass composition and tissue distribution in beef cattle using ultrasound scanning at the start and/or end of the finishing period. Livest. Sci. 131, 193-202.

Leeds, T.D., Mousel, M.R., Notter, D.R., Zerby, H.N., Mollet, C.A., Lewis, G.S., 2008. Bmode, real-time ultrasound for estimating carcass measures in live sheep: accuracy of ultrasound measures and their relationships with carcass yield and value. J. Anim. Sci. 86, 3203-3214.

Maroco, J., 2007. 'Análise Estatística - Com Utilização do SPSS'. (Ed. Sílabo, Lda., $3^{\text {a }}$ edição, Lisboa).

Moeller, S.J., Christian, L.L., 1998. Evaluation of the accuracy of real-time ultrasonic measurements of backfat and loin muscle area in swine using multiple statistical analysis procedures. J. Anim. Sci. 76, 2503-2514.

Orman, A., Caliskan, G.U., Dikmen, S., 2010. The assessment of carcass traits of Awassi lambs by real-time ultrasound at different body weight and sexes. J. Anim. Sci. 88 (10), 3428-3438.

Peres, A.M., Dias, L.G., Joy, M., Teixeira, A., 2010. Assessment of goat fat depots using ultrasound technology and multiple multivariate prediction models. J. Anim. Sci. 88, $572-580$.

Ribeiro, F.R.B., Tedeschi, L.O., Stouffer, J.R., Carstens, G.E., 2008. Technical note: a novel technique to assess internal body fat of cattle by using real-time ultrasound. J. Anim. Sci. 86 (3), 763-767.

Ripoll, G., Joy, M., Rodriguez, J.A., Sanz, A., Teixeira, A., 2009. Estimation of light lamb carcass composition by in vivo real-time ultrasonography at four anatomical locations. J. Anim. Sci. 87, 1455-1463.

Ripoll, G., Joy, M., Sanz, A., 2010. Estimation of carcass composition by ultrasound measurements in 4 anatomical locations of 3 commercial categories of lamb. J. Anim. Sci. 88, 3409-3418.

Scholz, A.M., Bünger, L., Kongsro, J., Baulain, U., Mitchell, A.D., 2015. Non-invasive methods for the determination of body and carcass composition in livestock: dualenergy X-ray absorptiometry, computed tomography, magnetic resonance imaging and ultrasound: invited review. Animal 9 (7), 1-15.

Silva, S.R., Afonso, J.J., Santos, V.A., Monteiro, A., Guedes, C.M., Azevedo, J.M.T., Dias da-Silva, A., 2006. In vivo estimation of sheep carcass composition using real time ultrasound with two probes of 5 and $75 \mathrm{MHz}$ and image analysis. J. Anim. Sci. 84, 3433-3439.

Silva, S.R., Santos, V.C., Batista, A.C.S., Jorge, A.M., Guedes, C.M., Teixeira, A., 2014. The ability of video image analysis (VIA) to predict carcass composition and cut yields of light lamb carcasses, FAIM III, Taastrup, Denmark, Conference Paper. pp. 37.

Silva, S.R., Afonso, J., Monteiro, A., Morais, R., Cabo, A., Batista, A.C., Guedes, C.M., Teixeira, A., 2017. Application of bioelectrical impedance analysis in prediction of light kid carcass and muscle chemical composition. Animal 17, 1-7.

Silva, S.R., Guedes, C., Rodrigues, S., Teixeira, A., 2020. Non-destructive imaging and spectroscopic techniques for assessment of carcass and meat quality in sheep and goats: a review. Foods 9, 1074.

Stevens, A., Ramirez-Lopez, L., 2013. An introduction to the prospectr package, $\mathrm{R}$ package Vignette [Online]. Available at: https://cran.r-project.org/web/packages/ prospectr/vignettes/prospectr-intro.pdf.

Stouffer, J.R., 2004. History of ultrasound in animal science. J. Ultrasound Med. 23, 577-584.

Szabo Cs, Babinszky L., Verstegen, M.W.A., Vangen, O., Jansman, A.J.M., Kanis, E., 1999. The application of digital imaging techniques in the vivo estimation of the body composition of pigs: a review. Livest. Prod. Sci. 60, 1-11.

Teixeira, A., 2008. Basic composition: rapid methodologies. In: Nolletl, L., Toldra, F. (Eds.), Handbook of muscle foods analysis. CRC Press. Taylor and Francis Group, pp. 291-314.

Teixeira, A., 2015. Ultrasound analysis. In: Nollet, L., Toldra, F. (Eds.), Handbook of Food Analysis. CRC Press. Taylor and Francis Group, pp. 673-686.

Teixeira, A., Delfa, R., Colomer-Rocher, F., 1989. Relationships between fat depots and body condition score or tail fatness in the rasa Aragonesa breed. Anim. Sci. 49 (2), 275-280.

Teixeira, A., Azevedo, J., Delfa, R., Morand-Fehr, P., Costa, C., 1995. Growth and development of Serrana kids from Natural Park of Montesinho (NE of Portugal). Small Rumin. Res, 16, 263-270.

Teixeira, A., Matos, S., Rodrigues, S., Delfa, R., Cadavez, V., 2006. In vivo estimation of lamb carcass composition by real-time ultrasonography. Meat Sci. 74, 289-295.

Teixeira, A., Joy, M., Delfa, R., 2008. In vivo estimation of goat carcass composition and body fat partition by real-time ultrasonography. J. Anim. Sci. 86, 2369-2376.

Teixeira, A., Silva, S., Rodrigues, S., 2019. Chapter six - advances in sheep and goat meat products research. Adv. Food Nutr. Res. 87, 305-370.

Thwaites, C.J., 1984. Ultrasonic estimation of carcass composition. Aust. Meat Res. Comm, 47, 1-31. 